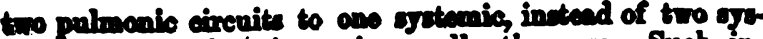
thic to ane pulmonic, as is ususlly the case. Buch intences hare been recorded by Meckel and Corvient; but the symptoms of the cases are not described by those suthors. However, in the Dublin Journal for 1848 , a similar case is inpplied by Dr. Mayne, and all the particulars are stated.

The symptoms presented by the patient were strictly peculiar, and lasted for years. Taking them all in all, they pere identical with those which we all feel during intense heat of the westher, when the hot medium surrounding us will not carry off all the caloric which our bodies are prepared to radiate. The patient, says Dr. Mayne, had the greatest dislike to change her position. She suffered from dyspncea and palpitation of the heart on taking exercise : and one remarkable symptom was constant sneating, which, unlike that of hectic, was unceasing; 80 that her linen was at all times saturated with moisture. She had also but little desire for nourishment. The bowels were torpid, and she seemed as incapable of mental as of bodily exertion. She died suddenly, after taking an unusual amount of exercise.

As cases of cyanosis, where the blood passes from the right to the left side of the heart without being properly arterialised, give evidence of deficient calorification, and afford a negative proof that the amount of caloric generated in the body is in proportion to the free union of blood and atmosphere, the peculiar case related by Dr. Mayne gives affirmative proof of the same fact. The arterial blood, passing twice through the lungs, and diffused through the venous as well as through the arterial system, could not but produce those effects in the system described by Dr. Mayne-effects which all happen when renous blood becomes arterial in character, as it is in the tropics, and eren in the temperate zones, when the summer heat is very great. It is unfortunate that Dr. Mayne did not note the temperature of this woman's body; though I do not myself imagine that he would have found it much increased, becanse the profuse elimination from the skin would equalise the animal temperature, as it does in cases where the body is exposed to extraordinary heat, as in the experiments of Blagden and Fordyce, and in operations attendant on the drying of cloth-prints in the heated chambers of printing astablishments.

The last on the list of arguments for the chemical theory of animal heat has reference to anæsthesia. To enter fully into this argument, I should be obliged to go over the whole ground of debate, in relation to the modus operandi of narcotics in general; and should be forced to refer to Dr. Snow's theory of their modus operandi, and to use it as the basis of mine. After 80 full and able a description of the theory as that which its originator has given at the last Physiological Meeting of the Medical Society, and in his published essays, I feel that but little is required of me. That all the agents which have the property of producing angsthesia have the property also of stopping oxidation, and even of suspending ordinary combustion, must be admitted by every experimentalist, and may be proven by any one who does not grudge the time and labour necessary for making a few simple experiments. It is curious, moreover, that those agents which possess the greatest power in suspending ordinary combustion, exert the quickest influence on the animal body in producing ansesthesia.

If then, these truths are taken, and are coupled with Dr. Snow's carefully proven facts, that, during anæsthesia, all the products of respiration, viz., those expired from the lunge, those formed in the blood, and those eliminated by the kidneys, are diminished; and that, in proportion to this diminution - undeniably a sign of decreased chemical action -the temperature of the body invariably falls; if, I say, these facts are all borne in mind, I think the inference is clear and just, that the decreased temperature in the anæasthetic state is an effect of decreased chemical action.

When I sat down for the first time to the writing of this paper, I did 80 with the intention of introducing into it the histories of several experiments relating to the animal tempernture which I have been conducting with considerable pare during the part three years; but, on entering upon the

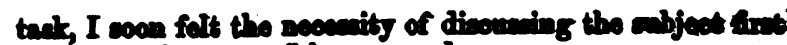
in a general way, as I have now done.

For future occacions, therefore, I recerre manj opecinl points, wuch as the extent of the oxidising procees in the production of animal combustion; the union of oxysen with other blood constituents than carbon and hydrogen; the parts of the body in which the chemical changes occur that lead to the evolution of heat; the question of the uses of the animal temperature,-Is it a mere chemical reault running off from the body as fast as it is formed; and doing nothing? or is it, in truth, an animating, if not the animating principle?

These, and indeed many other questions connected with the animal temperature, commend themselves much to all who desire earnestly to see a sound practice of medicine based on a sound physiology. I believe there is no scientific medical subject in which practical, pathological, and physiological observations fuse more kindly than in this: and that, if we could brush away difficulties, and seo clearly the full meaning of the calor vitalis, we should view with unclouded sight many of the most obscure points in pathology, and derive much solid information on the treatment of every disease.

Mortlake, January 1854.

$\stackrel{-}{-}$

\section{LECTURES, DELIVERED AT THE LOCK HOSPITAL, LONDON.}

By HENRY LEE, Esq., F.R.C.S., Surgeon to the Hospital. No. III. (Nov. 22ND, 1853.)

OX THE MEANS BY WHICH THE STPHIMITIC POIBON ENTRRB THE CONSTITUTION.

Physronogists have usually recognised three modes by which absorption may take place :-

I. That by which foreign substances find their way directly into the blood through the coats of the blood vessels. This mode of absorption occurs when poisonous substances are applied to an internal vascular and membranous surface, or when they are introduced into a wound, or when they are forced through the epidermis by friction on the surface of the body.

II. The absorption of the chyle from the mucous surface of the intestines by the lacteals. When the chyle is thus absorbed, it has been supposed by many physiologists that various other substances may be taken with it into the system.

III. Absorption by the lymphatics. This is supposed to occur either in the ordinary growth and renovation of the frame, or when parts are removed and not at the same time replaced, as in ulceration.

To these three modes of absorption, by all of which extraneous substances have been supposed to enter the circulation, we may add a fourth means by which the system may be influenced by the action of some kinds of poison : -namely, that in which a direct local effect is produced upon the nerres of a part, and through them upon the brain, (and consequently upon other organs), without the poisonous material being taken into the blood.

Examples of this mode of the action of poisons are afforded by the effects of the juice of the leaves of the aconite, and of the infusion of tobacco, as illustrated in some of Sir B. Brodie's physiological experiments.

In all those instances the action of the poison commences immediately upon its application.

But there are a very remarkable, and, to us, most interesting class of cases in which this does not happen. In these a certain interval must elapse, and a certain morbid process must be gone through before the poisons can produce their specific actions upon the general system. They are, therefore, with peculiar propriety ranked among the morbid poisons.

The deleterious materials of which these escentially confist, or the secretions in which they are contained, mas be applied to the living body in anj quantity, and for ans 
length of time; and unless the process, which is peculiar to each of them respectively, be gone through, their cherecteristic effects will not be produced upon the constitution.

If the particular action by which they individually enter the system be disturbed or interfered with-so as not to be carried out - other results may follow, but their general influence will not be experienced. If the first step in the process be defective, so will be its natural consequences.

The knowledge which we thus obtain furnishes us with a most important power; for by modifying or altering the morbid process by which a poison enters the system, we may, under certain circumstances, modify or control its effects.

I purpose now to consider how far the action of the syphilitic poison upon the constitution may be thus modified or controlled, by the kind of action which is produced when it is first applied to the living tissues of the body. For this purpose it is requisite to consider what the morbid actions really are by which the syphilitic poison gains an entrance; and what the natural processes are by which it joins itself to, and becomes as it were incorporated with, the living being.

The opinions of Hunter on the absorption of the syphilitic poison have given a bias to all subsequent reasonings upon the subject; and the theories based upon his experiments are very generally received even up to the present time. He demonstrated, as he believed, that the fymphatic vessels were the true absorbents; and he concluded from his discovery that they were the only absorbents in the system.

The experiments upon which his idea was founded deserve attention. Assisted by his brother and several other eminent medical men, he confined some warm milk in a portion of small intestine; and having tied the artery and vein which supplied the intestine, he saw, as he believed, the lacteals of the part presently become filled with the white milk. Upon puncturing the vein upon the distal side of the ligature, it was soon (by pressure of the finger), emptied of its blood. No white fluid could, during the continuance of the operation, be found in the vein. Upon a repetition of the experiment, in which the circulation through the mesenteric vessels was left free, the blood in the vein was carefully examined and compared with that in the neighbouring veins, but it was found not to be light coloured, nor milky, nor could any difference whatever be detected in it. It was found that even by firm pressure, which was continued until the intestine burst, the milky fluid could not be made to pass into the reins.

In another animal some thin starch, coloured with indigo, was introduced into the small intestine, and the lacteals were soon afterwards seen of a fine blue colour. A vein in this part of the mesentery was opened, and the blood which flowed was allowed to separate into coagulum and serum. The next day the serum had not the least blueish cast.

An injecting pipe was then fixed in an artery of the mesentery, where the intestine was filled with blue starch, and all communications both in the mesentery and intestine were closed, with the exception of the vein corresponding with the artery. Warm milk was now injected until it returned by the vein. This was continued until all the blood was washed away, and the vein returned a bright white milk. The milk thus circulating through the intestine containing the blue starch, was not in any degree changed in colour.

In a third animal, some musk in warm water was confined in a portion of the intestine; after waiting a little time, some of the lacteals of the part were opened with a lancet, and some of the watery fluid which they contained was received into a small spoon. This was found to smell strongly of musk. Some blood received into a clean spoon from one of the veins of the same part, had not the least smell of musk.

From these and similar experiments, Hunter arrived at the inference, which must have appeared one of the greatest discoreries of his age, that the vetns do not absorb in the human body. It necessarily followed from this that the lymphatics were to be considered as the only absorbents; and this is stated by Hunter to be the fact, in his work on the venereal disease.

If the lymphatics were the only absorbents, they must of necessity have been looked upon as the only channels through which poisons could, under ordinary circumstances, enter the system; and accordingly we find Hunter asserting that the venereal matter is taken up by the absorbents of the part in which it is placed, and carried along the absorbent vessels to the common circulation. (pp. 256257).

This view, deriving as it does such an apparent confirmation, with regard tc the venereal disease, from the frequent occurrence in it of inflamed lymphatic glands, has been adopted, with more or less modification, by almost all subsequent writers.

The accuracy of the experiments upon which Hunter based his theory have, however, justly been doubted. by other physiologists; but the theory itself has hitherto, strange to say, scarcely been questioned.

MM. Tiedemann and Gmelin, after mixing various substances, which might easily be detected, with the food of animals, not unfrequently found unequivocal traces of these substances in the venous blood and urine, whilst it. was only in a very few instances that traces of them could be discovered in the chyle.

In repeating Hunter's experiments, Mayo found that half an hour after a solution of starch and indigo had been placed in the carity of the intestine, the lacteals appeared of a clear blue colour, and those present were for a time. satisfied that the indigo had been absorbed. But upon placing a sheet of white paper behind the mesentery, it was found that the blue tinge disappeared. On removing the white paper the vessels reassumed their blue colour. It became, therefore, evident that the blue tinge was the natural colour of the empty lacteals; - that while they continued to absorb the chyle they were white, but that as soon as they were simply empty, they appeared blue.

Thus a repetition of the Hunterian experiments rather tends to prove that the function of the lacteals is limited to the absorption of chyle: and that the lymphatics are not the only absorbents, appears most conclusively demonstrated by the experiments of MM. Magendie and Ségalas. M. Ségalas varied Hunter's experiment in the following manner:-a fold of small intestine was drawn out of a wound in the belly of a dog; all the blood-ressels passing to and from it were tied, with the exception of one artery; a vein punctured upon the mesentery allowed the blood to escape, which would otherwise have stagnated in the part. The lacteal vessels and nerves were left entire. The fold of intestine was then tied at both extremities, and an aqueous solution of the alcoholic extract of nux vomica was poured into it. During the hour which followed, the poison produced no symptoms. The ligatures being then removed from one of the veins, the blood was allowed to return in the natural course of its circulation. In six minutes from this time, the poison took effect. The experiments of M. Magendic illustrating the same point are well known.

The thigh of a dog was separated by M. Magendie from the body, by a division of every part, with the exception of the artery and vein ; into each of these vessels a quill was introduced, and tied by two ligatures; between these ligatures the vessels were divided, and thus all communication was cut off between the body and the limb, except that which was maintained by the circulation of the blood. Two grains of the upas tieute were then inserted into $a$ wound in the foot of the separated limb. In about four minutes the poison manifested its effects upon the system.

From these and other facts, it appears certain that Hunter's idea of the lymphatics being the only absorbents is incorrect; and we are thence naturally led to the consideration of the value of the theory which was based upon that notion. 
A careful obeerration of the origin of sphilitic croo cins will show that the disensed action to which the pison gives rise on its primary application, is not always the ame. The differences observable may bo traced either in the natural course of the disease, or by means of artifein inoculation.

In one class of cases as observed in practice, the secrotion from the infected part will, in the early stage of the disease, consist of a thin serous fluid more or less turbid and the parts immediately involved will become indurated in a very peculiar and characteristic manner.

In a second class, the discharge will at the same period be more discoloured, and the parts affected will present a ragged and uneven surface. There will here be none of the peculiar and characteristic induration above mentioned.

In a third class, the socretion will from the first consist of well formed pus. The surface from which it proceeds may be even and regular, and there will be no peculiar induration, except that which may result from the granulations of the exposed surface.

In a fourth class, the part infected passes at once into mortification, or into that modification of it which has been called phagedana.

After artificial inoculation these four kinds of affection may likewise be distinguished; but, as will hereafter be more fully explained, (from the fact of inoculation having been almost exclusively practised on those whose systems were already under the influence of the syphilitic poison), one or two of these forms of disease have been much more commonly observed than the others.

We may, however, distinctly observe that in some cases after inoculation, no pus is secreted before the fourth or fifth day. Up to this time the secretion is a fluid, transparent at first, but becoming gradually more turbid. As it becomes purulent, the subjacent tissues become infiltrated with plastic lymph, which gives to the touch the sensation of certain forms of cartilage.

At other times after inoculation, we find that the surface of the part will be covered by a crust of different shades of brown or yellow; and when this is removed, that a ragged irregular surface will present itself.

Again, in another class of cases the inoculation will present, as early as the following day, a well formed pustule. The secretion will be quite white, and entirely different in character from that observed at the same period in the first above-mentioned class of cases.

Finally, we may have a phagedenic or sloughing sore as the result of inoculation, an effect often purposely produced by the application of caustie. In none of the three last descriptions of cases have we the characteristic induration observed in the first.

In immediate connexion with these different kinds of primary affection, I have to observe-

I. That in practice a sore which presents from the first a ragged and uneven surface will, with tolerable certainty, be accompanied by an inflammatory bubo; and that this bubo will very generally suppurate be the treatment what it may.

Ir. That in the experiments which have been so lavishly performed by means of inoculation (in attempting to induce that condition of the system known or imagined as a state of syphilisation and for other purposes), the occurrence of any secondary results from those inoculations has been ahmost unknown.

Irr. As bearing directly and practically upon the theory of the absorption of the syphilitic poison, an extensive obcervation of cases will be found to establish the two following very important points, viz.: that in those cases where the irritation of the lymphatic glands is the greatest, we have very seldom indeed any sccondary syphilitic affection; and that in the best marked cases of general infection we as rarely find that the constitutional disease has been preceded by inflammation of the lymphatics. These latter circumstances are directly opposed to the idea of the grtem being contaminated throagh the absorbent vesels: where these are most affected, the system generally

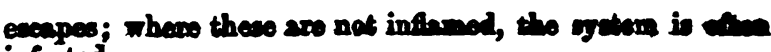
infected.

This subject has been very imperfectly illustental by the physiologists of the present day, and even those who lape written expresely upon oyphilitic affections have generally been satisfied with recording the facts which they hase abserved relating to it, without attempting to explain them.

Something more satisfactory, I am inclined to bolieve, may be arrived at, by an attentive consideration of the earliest stages of the morbid processes which are involved in the absorption of the syphilitic poison.

The great author of this mode of investigating disease hae prefaced his treatise on venereal affections with the following remarks which here find their appropriate place, and which, as I believe, have not received the amount of attention which they deserve, and have not been applied as they might have been to the illustration of our present subject. "No two actions", says Hunter, "can take place in the same constitution, nor in the same part, at one and the same time. No two different fevers can exist in the same constitution, nor two local diseases in the same part at the same time." It might appear strange to any one who had not considered the subject in its physiological relations, that wach ideas should occupy so prominent a position in Hunter's work on the venereal disease, and that they should be dwelt upon in this rather than in any other of his writings. They are, nevertheless, I believe, the principles upon which much that is apparently obscure in relation to this diseace may bo explained. They afford a remarkable instance of that intuitive insight so peculiar to our great physiologist, by which comprehensive general ideas are appreciated in their extent and simplicity, even where their application to particular details may not have been traced.

For truth and clearness, the description of a primary syphilitic ulcer has not been excelled since Hunter's time; "a chancre", he says, "has commonly a thickened base, and although in some sores the inflammation spreads much farther, yet the specific inflammation is confined to this base." This specific action in which the arteries throw out coagulable lymph, depends, according to the Hunterian nomenclature, upon adhesive inflammation. The action by which parts are removed is called the ulcerative inflamenation; that by which pus is formed, the suppurative inflammation.

These three effects of inflammation Hunter regands as distinct actions, and therefore incapable of being produced in the same part at the same time. Now that which in peculiarly characteristic of the syphilitic infection of a part is a specific adhesive inflammation. This has no necessary connexion at all with either ulceration or suppuration, and indeed, according to the Hunterian doctrine, it is incompatible with either of them. Of all the kinds of inflammation to which the contact of syphilitic matter gives rise, this alone can be distinctly and certainly associated with the occurrence of secondary symptoms.

It is true that we almost always find that a part affected with syphilitic induration also suppurates, or ulcerates upon its surface; and in the more advanced stages of the disease we often see that all the parts which have been indurated pass into suppuration or ulceration. In the former case, the adhesive and suppurative inflammations affect different parts (although in close proximity to each other); in the latter, these distinet actions affect the same parts but at different times.

An action commenced in a part will continue until the cause determining it ceases; or until it is superseded by some more powerful action. If, therefore, the ulcerative or suppurative inflammation be set up by venereal infection, it will continue until the poison has expended its influence, or until the part is attacked by mortification, or influenced by some other cause sufficiently powerful to suparsede the original action. Hence it fallows that if a venoreal sose in its origin is affected with either the suppurative or the ulcerative inflammation, it is most mnlikely subeoquently to become indurated.

A mont important distination hare eries between then 
enees which in their origin are socompanied by specific adhesive inflammation, horrever limited in extent, and those which from the first suppurate, ulcerste, or slough. In the first class the poison will, with tolerable certainty, affect the system, unless prevented by medical treatment or the influence of some peculiarity, or some other disease. In the latter, the local affection will never, I believe, be followed by constitutional syphilis. Ulceration and suppuration, like mortification, destroy the vitality of the parts which they attack, although in a more gradual manner, and as the syphilitic virus requires a living nidus and a certain given space of time for its development, it is destroyed in these actions before it becomes, in the process of growth, taken into the system.

Thus practically we may say, that if a suspicious sore is from the first affected with ulcerative inflammation, or if from the first there be a free secretion of well formed pus, or if at the same period it should slough either naturally or from medical applications, the existence of the syphilitic virus will cease with that of the parts which it has infected. The disease, as far as its specific characters are concerned, will be a local one.

From what has already been said, it may be inferred that ulcerative inflammation is as incompatible in the same part at the same time with suppurative inflammation as it is with adhesive inflammation; and, in perfect accordance with this, we find practically that when an infected sore suppurates very freely from the first, or sloughs, that there is seldom any affection of the lymphatic system. But that, on the other hand, when ulcerative inflammation is early established, characterised by the ragged and uneven surface of the sure, the absorbent glands become suddenly and violently inflamed.

In these cases, we may trace in the most satisfactory manner the progress of the syphilitic poison along the absorbent vessels as far as the first lymphatic gland with which it comes in contact. In any part of this course the poison may be arrested, and may there produce a fresh syphilitic ulcer; and that the absorbent glands into which these lymphatics empty themselves may become affected, we have daily proof from the secretions of these glands being themselves inoculable. Up to this point, then, we have uuequirocal evidence of the presence of the syphilitic poison, and of its power of contaminating fresh parts. Beyond this, the poison can no longer be traced. The fluids in which it was before contained now neither possess the power of irritating the vessels through which they pass, nor, when extravasated from these vessels, of infecting other structures. Here, then, some wonderful change is produced. The specific characters of the poison can no longer be detected either by its morbid effects, or by inoculation. Even Hunter noticed this circumstance. He says, "we never find the lymphatic vessels or glands that are second in order, affected". And he remarks that when the disease has been contracted from a cut upon the finger, he has seen the bubo come on a little above the bend of the arm, upon the inside of the biceps muscle. In such instances no bubo has formed in the armpit, the most common place for glands to be affected by absorption.

Neither observation nor experiment, then, afford any proof that the syphilitic virus is conveyed, as such, through the absorbent glands; all the direct evidence which we have points to an opposite conclusion. The particles in which the poison resides here undergo some change, become perhaps disintegrated, and in that process the existence of the poison appears to cease.

The evidence upon which it has been assumed that the syphilitic poison enters the system through the absorbent vessels must, therefore, be regarded as most inconclusive. Arrived at the first lymphatic gland which it meets, and there undergoing some change, it becomes incapable any longer of producing, either locally or constitutionally, its specific effects; and we cannot therefore admit that this is the way in which the system bocomes infected. It will coubtless be asked, if a primary syphilitic affection will gire rise to a bubo which itself mas be prored by inoeulation to be syphilitic, how it is that this bubo does not become a fresh source of infection? How is it that the virus may not be absorbed from the parts thus affected, so as to produce a second bubo? and how is it that the poison from this source may not be received into the system by some other means? The answer to this question is twofold. Practically, neither of these circumstances occur; theoretically, the supposed difficulty is met by a consideration of the principles already laid down. During the time that the fluids impregnated with the poison are contained in the absorbent vessels, the poison can of course exercise its influence only upon those ressels. Whenever it does so, it gives rise to inflammation, which, as it depends upon ulcerative inflammation in another part, will probably here be of the same character. As soon as an absorbent vessel or gland is thus attacked, the cellular tissuc around it will become also inflamed, and will suppurate. All experiments have gone to prove that the pus formed on the outside of a lymphatic vessel is different in character to the fluid derived from the gland itself. The latter is inoculable; the former not. The action by which the parts around an affected lymphatic participate in the disease is, therefore, simple suppurative inflammation, which we have before considered as a process not capable of communicating the syphilitic disease to the general system. As, therefore, the contaminated fluid in a lymphatic vessel cannot pass unchanged through the absorbent glands, and can find its way to the surface only through tissues in a state of suppuration, in neither case are its poisonous effects communicated to other parts. In the action by which the poison contained in an absorbent gland is eliminated by suppuration, the whole surface involved may become inoculated by the syphilitic matter; but the inflammation being in its origin of the suppuratire kind, the original action is not by this fresh inoculation altered in its character. The process, once commenced, is continued, and, during its continuance, is incompatible, as we have seen, with specific adhesive inflammation, which, according to the view now taken, is alone capable of all the kinds of inflammation, of communicating general syphilitic infection.

To these remarks I may add, that experiment and observation concur in proving that, every time that a fresh inoculation takes place in the same individual from the same original source, the effects of the poison will show themselves with less severity.

It is not intended by anything that is here stated to imply that vitiated fluids may not enter the circulation through the absorbent system, even when the morbid process which gives rise to their formation has had its origin in the venereal disease. Well marked cases from time to time present themselves, in which the lymphatic vessels, the absorbent glands, and even the thoracic duct, are found distended with puriform or sanguinolent fluid. Such diseased products poured into the circulation must necessarily have a deleterious influence upon the constitution. They may gire rise occasionally to eruptions upon the skin, which may more or less resemble true syphilitic affections. These eruptions usually appear before the primary affection to which they may be attributed subsides. They often disappear of their own accord, and do not generally recur. Many of the diseases which, from Abernethy's time to the present, have been described under the titles of " diseases resembling syphilis", "a mild form of secondary symptoms", etc., I have little doubt, may be included in this class. They may depend, as I have said, upon the absorption of inflammatory products resulting from local venereal infection, but I cannot regard them as arising from the presence of the syphilitic virus itself.

The poison of the viper, or the Woorara poison, or a solution of opium, may be introduced into the stomach of some animals without producing their poisonous effects; but it does not follow that the digestion of these substances would be followed by no disturbance in the system. In any of the cases mentioned, some disorder might arise, although not depending upon the specific effects of the poison. In any, 
- lite sction might be induced by subetances in themsolves poesesing no specific or poisonous qualities.

From experiments wich have been now varied in a thousand ways, and most unnecessarily ropested, it has been proved beyond a doubt that the syphilitic poison may remain in contact with an abraded surface, or may be inserted benesth the cuticle, and allowed to remain there for three, four, or even five days, and no absorption may occur. If, during this time, an inflammatory action is set up, which is incompatible with specific adhesive inflammation, no general infection will follow. If, for instance, the part is made to slough by the application of caustic, as soon as the slough separates, a simple sore alone will remain. From this, it is ovident that a certain time must elapse after the application of the syphilitic poison before any absorption can take place. This requisite period of incubation it is that secures the system against infection in cases where, from the first, ulcerative or suppurative inflammation has taken place. A part, in the course of being contaminated, becomes by these processes dissolved or removed before the act of absorption can be completed. Fresh parts, which may continue to be attacked, are destroyed before they can act as the channels of infection to the constitution. Hence occasionally arise those extensive local intractable ulcerations which have received the name of serpiginous or creeping sores. These, howerer formidable they may be as local diseases, do not give rise to secondary syphilitic symptoms.

We have here the secret of the absence of constitutional results after artificial inoculation in the numerous experiments that have been made on syphalisation. These experiments have been tried only on those whose systems were already under the influence of the poison; and it appears to be a very general law that, in such cases, any fresh inoculations pass rapidly into suppuration, or ulceration-processes, as I have said, incompatible with the specific and infecting kind of inflammation. Where several artificial inoculations have been performed, and these inoculations have at once suppurated, the circumstances are in many respects similar to those in which any patient would be placed if he had a suppurating bubo. Many small spots of suppuration, and one large spot, I conceive to be nearly equivalent to each other; in neither case is the poison absorbed from these secondary inoculations. This circumstance it doubtless is that has given some of our continental brethren the idea that repcated inoculation may be a preventive against future syphilitic infection.

It is, I believe, undoubtedly true that, while a syphilitic sore is undergoing ulceration or suppuration, any similar affection arising from fresh inoculation will do the same; and it is quite possible that this tendency may be kept up by repeated inoculation for an indefinite time. During this period, no fresh absorption of syphilitic poison will take place. The same immunity is afforded by a suppurating bubo during its continuance, and perhaps for a considerable time after it has healed. But, in either case, allow an interval to elapse during which this tendency to ulceration or to suppuration shall have worn itself out, and the system will aguin become subject to genuine syphilitic infection, and be again liable to fresh forms of secondary disease.

From all the observations which have now been made, I conclude that the idea originated by Hunter, and received by his followers, that the syphilitic poison is taken into the system by the lymphatic vessels, is, to say the least, entirely devoid of proof. The real way in which the system does become infected may, I beliere, be traced in another and much more satisfactory manner.

When syphilitic inoculation takes place in a healthy person, and the regular course of the disease is not interfered with, $t$ wo distinct processes may be recognised; one, that by which the affected tissues become infiltrated with lymph; the other, by which this effused matter is removed. This latter result may be accomplished by sloughing, by ulceration, in the natural process of growth, or by different modifications of these. But, beyond the parts immediately involred in these processes, other actions are going on of a more cubtle nature, and not so easily appreciated by our senses.
In the absence of more positive knowledge, , Te mas ascribe these to the molecular changes in the nutrition of the surrounding parts. That such actions are in active operation beyond the parts where any visible or sensible change has taken place, may be readily demonstrated, although we may be unable to define their exact nature. Were this not the case, we should have nothing to do in the case of a primary syphilitic sore but entirely to remove the ulcerated and indurated tissues, and the disease would, as far as the part is concerned, be at an end. Experience proves that such is very far from being the case. When a syphilitic sore is removed by excision, as may readily be done when it is situated on the extremity of the prepuce, the cut surface will. in a few days take on the specific action. This I have verified even when the greatest care has been taken not to allow any other matter from the chancre to come into contact with the cut surface. Such an action taking place in a part apparently healthy, at some little distance from the original sore, presupposes some antecedent change in the original sore, presupposes some a change produced by the infecting poison, but not capable of being appreciated solong as the diseased action had its development in its original situation. As soon, however, as the first centre of the morbid action is remored, a similar disease is induced upon the neighbouring cut surface. The observation of such cases demonstrates the existence of a subtle morbid process beyond the parts at first sensibly affected, and necessarily producing some change in their nutrition.

It appears under these circumstances much more in accordance with that which is known to happen in the case of the absorption of other poisons, to suppose that the blood circulating through the tissues in which these morbid actions are going on is directly influenced, than to refer the symptoms to the passage of the poison primarily through the absorbent system. When the constitution becomes affected in consequence of the inoculation of the vaccine or the variolous poisons, the lymphatic glands appear certainly to perform no essential part of the process. Few indeed have thought it necessary to inroke the aid of the absorbent system to account for the action of these poisons upon the animal economy; and I believe that it is equally unnecessary in the case of the poison of syphilis.

At present, I have only considered the different kinds of inflammatory affections produced by syphilitic contagion. The existence of similar non-inflammatory modes of infection (such as the absorption of the syphilitic virus without. any change of structure in the parts to which it is applied, or the production by some means of tubercles or excrescences not having an inflammatory origin) form a subject for separate consideration.

\section{EXPERIMENTAL INQUIRY INTO THE EFFECTS OF INJECTING PUS INTO THE VEINS OF ANIMALS.}

\section{By JOSEPH SAMPSON GAMGEE, Esq.}

Froy the brief consideration of my experimental inquiry into the effects of injecting pus into the veins of animals, published by Mr. Henry Lee in the Association MedicaI JodRNAL for Dec. 23rd, it is evident that he adheres as firmly as ever to the doctrine which he propounded in 1850 , and that he has been in no degree impressed with my objections to it as being of much weight. He tacitly admits, indeed, that I have exposed the fallacy of one set of experiments on which he founded it, but he "cannot for a moment allow (as I appear to have inferred) that the conclusions arrived at depend upon the accuracy of any one set of experiments": but I anticipate that, however sanguine be Mr. Lee's confidence in his principles and conclusions, he will find it difficult to hold and inculcate them, unless he can maintain intact the experimental basis upon which he founded them. The matter is essentially one of experience; and if Mr. Iree's conclusions can be proved to be the result 